\title{
Assisted Work Distillation
}

\author{
Benjamin Morris, ${ }^{*}$ Ludovico Lami, ${ }^{\dagger}$ and Gerardo Adesso ${ }^{\ddagger}$ \\ School of Mathematical Sciences and Centre for the Mathematics and Theoretical Physics of Quantum Non-Equilibrium Systems, \\ University of Nottingham, University Park, Nottingham NG7 2RD, United Kingdom
}

(Received 19 December 2018; revised manuscript received 8 February 2019; published 2 April 2019)

\begin{abstract}
We study the process of assisted work distillation. This scenario arises when two parties share a bipartite quantum state $\rho_{A B}$ and their task is to locally distill the optimal amount of work when one party is restricted to thermal operations, whereas the other can perform general quantum operations and they are allowed to communicate classically. We demonstrate that this question is intimately related to the distillation of classical and quantum correlations. In particular, we show that the advantage of one party performing global measurements over many copies of $\rho_{A B}$ is related to the nonadditivity of the entanglement of formation. We also show that there may exist work bound in the quantum correlations of the state that is only extractable under the wider class of local Gibbs-preserving operations.
\end{abstract}

DOI: 10.1103/PhysRevLett.122.130601

Introduction.-The recently conceived field of quantum thermodynamics represents a drive to understand the interplay of the two fundamental theories of thermodynamics and quantum mechanics. Scientists from various disciplines such as open quantum systems [1], stochastic thermodynamics [2], and information theory [3] are utilizing their respective tools to answer these fundamental questions. In particular, recent work [4,5] has demonstrated that thermodynamics may be understood from a resourcetheoretic perspective, allowing researchers to investigate thermodynamic transformations in a quantum information setting.

In this Letter, we investigate the task of assisted work distillation, see Fig. 1. Here, the process of work distillation is intended in a resource theoretic framework to be the asymptotic distillation of reference states with energy but no entropy by means of thermal operations, meaning that the distillable (or extractable) work can be quantified by how distinguishable a quantum state is from a Gibbs equilibrium state [5]-for other definitions of work in quantum thermodynamics, see, e.g., Ref. [3]. In the assisted scenario, two parties, Alice $(A)$ and Bob $(B)$, share many copies of a bipartite state $\rho_{A B}$. Between them their goal is to maximize the quantity of distillable work on Bob's subsystem. Alice may perform arbitrary quantum operations on her subsystem, whereas Bob is restricted to thermal operations on his. By utilizing correlations within $\rho_{A B}$ and classical communication between the parties, we demonstrate key features of Bob's distillable work.

In particular, we characterize the set of shared states that allow for local work distillation. We also demonstrate that, for a protocol involving one-way communication between the parties, explicit expressions for the local distillable work, which we dub the work of assistance [in analogy with the entanglement of assistance [6]], can be derived both in the asymptotic and non-asymptotic scenarios. From these expressions we make use of two central results from quantum information theory to show that Alice performing global measurements over many copies of the shared state offers an explicit advantage over the case of single copy measurements. We also show that this advantage disappears when the initial state is pure.

In addition to the work of assistance, we also define the work of collaboration, defined as such to allow two-way communication between the parties and local Gibbs-preserving operations [7] on Bob's side. We show that by allowing this collaboration and the wider class of operations, the local distillable work can increase. We also demonstrate that for an initial pure state the work of collaboration may yield an increase in distillable work by an amount proportional to the entropy of Bob's subsystem $S\left(\rho_{B}\right)$, where $\rho_{B}=\operatorname{Tr}_{A}\left[\rho_{A B}\right]$.

It is important to consider the realm in which our results apply. Within the resource theoretic framework, it is typical

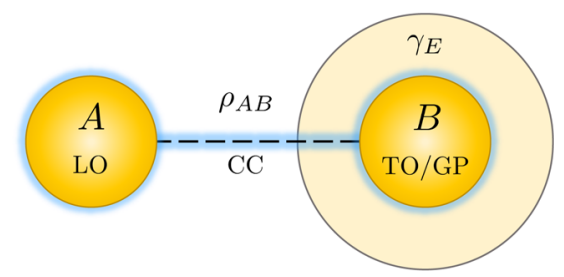

FIG. 1. We investigate distillation of work from a quantum system $B$ controlled by an observer, Bob, who is constrained to thermal operations or Gibbs-preserving operations, and is assisted by another party, Alice, who can perform arbitrary local operations on an ancillary system $A$ and communicate classically with Bob. The work of assistance and the work of collaboration are defined and related to the correlations in the state $\rho_{A B}$ shared by Alice and Bob. 
to consider resource inconvertibility in the asymptotic scenario. This is particularly pertinent for thermodynamics due to its equivalence to taking the thermodynamic limit, which suppresses the appearance of fluctuations.

Resource theories of thermodynamics. - We start by explicitly defining what is meant by the resource theory of thermal operations (TO). Originally introduced by Refs. [4,5], the allowed operations for a quantum system $S$ with Hilbert space $\mathcal{H}$ and Hamiltonian $H_{S}$ are the completely positive trace-preserving (CPTP) maps $\mathcal{E}: \mathcal{L}(\mathcal{H}) \rightarrow \mathcal{L}(\mathcal{H})$ of the form

$$
\mathcal{E}(\rho)=\operatorname{Tr}_{E}\left[U_{S E}\left(\rho_{S} \otimes \gamma_{E}\right) U_{S E}^{\dagger}\right],
$$

where $U_{S E}$ is an arbitrary unitary operation, acting jointly on the system $S$ and a reservoir $E$, that commutes with the global Hamiltonian $\left[U, H_{S} \otimes \mathbb{1}_{E}+\mathbb{1}_{S} \otimes H_{E}\right]=0$, and $\gamma=Z^{-1} e^{-\beta H}$ denotes the Gibbs thermal equilibrium state at inverse temperature $\beta$ and partition function $Z$. The joint unitary operations and partial trace define the free operations of the resource theory, whereas the Gibbs states define the free states. By explicitly accounting for the resources used, the TO framework provides a general setting within which to study thermodynamic transformations, in particular the distillation of work.

In this setting, following [8] we define the distillable work from a system $B$ in the state $\rho_{B}$ as the maximum number $R E$ such that the transformations $\rho_{B}^{\otimes n} \otimes$ $|0\rangle\left\langle\left. 0\right|_{P} ^{\otimes[R n]} \rightarrow \mid 1\right\rangle\left\langle\left. 1\right|_{P} ^{\otimes[R n]}\right.$ are possible with TO at background inverse temperature $\beta$ with asymptotically vanishing error. Here, referring to Eq. (1), we are considering a composite system $S$ that consists of the principal system $B$ with Hamiltonian $H_{B}$ and a qubit battery $P$ with Hamiltonian $H_{P}:=E|1\rangle\left\langle\left. 1\right|_{P}\right.$, where $E$ is a free parameter we are allowed to optimize over. In formula

$$
\begin{aligned}
W\left(\rho_{B}\right): & =\sup \{R E: \\
& \lim _{n \rightarrow \infty} \inf _{\Lambda \in \mathrm{TO}} \| \Lambda\left(\rho_{B}^{\otimes n} \otimes|0\rangle\left\langle\left. 0\right|_{P} ^{\otimes[R n]}\right)-|1\rangle\left\langle\left. 1\right|_{P} ^{\otimes[R n]} \|_{1}=0\right\} .\right.
\end{aligned}
$$

It follows from the main result of Ref. [5] [see Appendix A in Supplemental Material [9] for an explicit derivation] that the distillable work defined in Eq. (2) equals the change in free energy

$$
W\left(\rho_{B}\right) \equiv \Delta F\left(\rho_{B}\right)=\frac{1}{\beta} S\left(\rho_{B} \| \gamma_{B}\right),
$$

with $S(\rho \| \gamma)=\operatorname{Tr}(\rho \log \rho-\rho \log \gamma)$ being the relative entropy of athermality. Observe that $S(\rho \| \gamma)$ is monotonically nonincreasing under TO.

A larger class of operations are Gibbs-Preserving (GP) operations; these are CPTP maps $\Lambda$ that admit as their fixed point the Gibbs state at a given temperature, i.e., such that
$\Lambda\left(\gamma_{B}\right)=\gamma_{B}$. The motivation behind this alternative framework that regards GP operations as free operations for thermodynamics, is that any non-GP operation, $\Lambda(\gamma)=\sigma \neq \gamma$, could be used to extract an arbitrarily large amount of work from $\sigma^{\otimes n}$ as $n \rightarrow \infty$. It can be clearly seen from Eq. (1) that TO are a subset of GP, and the inclusion is known to be strict [7].

Work of assistance.-In this section, we consider the case where Alice and Bob have access to the shared state $\rho_{A B}$ and we allow one-way classical communication from Alice to Bob. This is similarly motivated as the recently studied "conditioned thermal operations" [18]. Alice, whom operations are unrestricted, may perform on her subsystem the positive operator-valued measurement (POVM) $\left\{\Pi_{A, i}\right\}$, whose associated probabilities are $p_{i}=\operatorname{Tr}\left[\rho_{A} \Pi_{A, i}\right]$, whereas Bob is restricted to TO. Alice performing her measurement and communicating the outcome to Bob results in him having access to the ensemble $\left\{p_{i}, \tilde{\rho}_{B, i}\right\}$, where

$$
\tilde{\rho}_{B, i}=\frac{1}{p_{i}} \operatorname{Tr}_{A}\left[\left(\Pi_{A, i} \otimes \mathbb{1}_{B}\right) \rho_{A B}\right] .
$$

In the scenario we consider, Alice's goal is to help Bob to distill as much work as possible. From this train of thoughts, we define our first quantity of interest, the work of assistance

$$
W_{a}^{B \mid A}\left(\rho_{A B}\right):=\max _{\left\{\Pi_{A, i}\right\}} \frac{1}{\beta} \sum_{i} p_{i} S\left(\tilde{\rho}_{B, i} \| \gamma_{B}\right),
$$

where the maximization is taken over the set of Alice's measurements (i.e., POVMs). Using convexity, we see that this quantity is lower bounded by $(1 / \beta) S\left(\rho_{B} \| \gamma_{B}\right)$, which of course means that being assisted by Alice is generally no worse than having no assistance at all. Moreover, as we show in Appendix B [9], all states $\rho_{A B}$ that exhibit some form of correlation, i.e., such that $\rho_{A B} \neq \rho_{A} \otimes \rho_{B}$ is not factorized, satisfy the strict inequality $W_{a}^{B \mid A}\left(\rho_{A B}\right)>$ $(1 / \beta) S\left(\rho_{B} \| \gamma_{B}\right)$, implying that there is an assisted protocol that helps Bob distilling more work. In particular, the states from which Bob can distill no work at all even in the assisted setting are simply products of the form $\Gamma_{A B}=\sigma_{A} \otimes \gamma_{B}$, from now on referred to as quantumthermal (QT) states; the same states have been found in the conditioned thermal operations setting [18].

In Appendix C [9], we show that $W_{a}^{B \mid A}$ can be written as

$$
W_{a}^{B \mid A}\left(\rho_{A B}\right)=\frac{1}{\beta}\left[S\left(\rho_{B} \| \gamma_{B}\right)+J^{\rightarrow}\left(\rho_{A B}\right)\right],
$$

where $J \rightarrow\left(\rho_{A B}\right)$ is the Henderson-Vedral [19] measure of classical correlations (with respect to measurements on Alice) defined as $J^{\rightarrow}\left(\rho_{A B}\right):=\max _{\left\{\Pi_{A, i}\right\}}\left[S\left(\rho_{B}\right)-\sum_{i} p_{i} S\left(\tilde{\rho}_{B, i}\right)\right]$. The result in Eq. (6) clearly separates the quantity of work 
distillable by Bob with or without the assistance of Alice. This is in agreement with a recent result in Ref. [20].

An important question to ask is whether this quantity of work changes if Alice is able to perform measurements over many copies of the shared initial state $\rho_{A B}$. In order to answer this question, we continue by defining the regularized work of assistance

$$
W_{a, \infty}^{B \mid A}\left(\rho_{A B}\right):=\lim _{n \rightarrow \infty} \frac{1}{n} W_{a}^{B \mid A}\left(\rho_{A B}^{\otimes n}\right) .
$$

In Appendix D [9], we show that the above quantity indeed yields the best achievable rate of work distillation in the case where the only allowed communication is from Alice to Bob. Although the regularization makes it hard to compute, the rhs of Eq. (7) can nonetheless be related to a quantifier known as distillable common randomness $C_{D}$, introduced in Ref. [19] as

$$
C_{D}^{\rightarrow}\left(\rho_{A B}\right)=\lim _{n \rightarrow \infty} \frac{1}{n} J^{\rightarrow}\left(\rho_{A B}^{\otimes n}\right),
$$

and then interpreted operationally in Ref. [21]. The operational interpretation of $C_{D}$ rests on protocols that extract from $n$ independent copies of $\rho_{A B}$ a total of $C$ maximally correlated classical bits via $R$ bits of noiseless classical communication between Alice and Bob with vanishing error. The quantity $C_{D}$ is thus defined as the maximum net gain $(C-R) / n$ in the limit $n \rightarrow \infty$. For a discussion from the thermodynamical point of view, see Ref. [22].

Using the definition in Eq. (8) and the fact that the relative entropy is additive, we can therefore write the regularized work of assistance as

$$
W_{a, \infty}^{B \mid A}\left(\rho_{A B}\right)=\frac{1}{\beta}\left[S\left(\rho_{B} \| \gamma_{B}\right)+C_{D}^{\rightarrow}\left(\rho_{A B}\right)\right],
$$

again clearly separating the quantity of distillable work with or without the assistance of Alice.

Upon defining the regularized version of $W_{a}^{B \mid A}\left(\rho_{A B}\right)$, we should ask whether giving Alice the ability to perform global measurements over many copies of the shared state $\rho_{A B}$ increases the average work that Bob can distill. In order to answer this question, we employ two fundamental results from the field of quantum information. On the one hand [[23], Theorem 1] states that

$$
\begin{aligned}
& E_{f}\left(\rho_{A^{\prime} B}\right)+J^{\rightarrow}\left(\rho_{A B}\right)=S\left(\rho_{B}\right), \\
& E_{C}\left(\rho_{A^{\prime} B}\right)+C_{D}\left(\rho_{A B}\right)=S\left(\rho_{B}\right),
\end{aligned}
$$

provided that $\rho_{A^{\prime} B}$ is the $A$ complement of $\rho_{A B}$; i.e., there exists a pure state extension $\rho_{A A^{\prime} B}$ that satisfies $\operatorname{Tr}_{A}\left[\rho_{A A^{\prime} B}\right]=$ $\rho_{A^{\prime} B}$ and $\operatorname{Tr}_{A^{\prime}}\left[\rho_{A A^{\prime} B}\right]=\rho_{A B}$. Here, $E_{f}\left(\rho_{A B}\right)$ stands for the entanglement of formation [24], while the entanglement cost is given by $E_{C}\left(\rho_{A B}\right)=\lim _{n \rightarrow \infty}(1 / n) E_{f}\left(\rho_{A B}^{\otimes n}\right)$, and quantifies the amount of Bell states needed to form $\rho_{A B}$ via local operations and classical communication in the asymptotic limit of many copies [25].

Substituting Eqs. (10) and (11) into Eqs. (6) and (9), respectively, allows us to write $W_{a}^{B \mid A}\left(\rho_{A B}\right)$ and $W_{a, \infty}^{B \mid A}\left(\rho_{A B}\right)$ in terms of these entanglement measures

$$
\begin{aligned}
W_{a}^{B \mid A}\left(\rho_{A B}\right) & =\frac{1}{\beta}\left[S\left(\rho_{B} \| \gamma_{B}\right)+S\left(\rho_{B}\right)-E_{f}\left(\rho_{A^{\prime} B}\right)\right], \\
W_{a, \infty}^{B \mid A}\left(\rho_{A B}\right) & =\frac{1}{\beta}\left[S\left(\rho_{B} \| \gamma_{B}\right)+S\left(\rho_{B}\right)-E_{C}\left(\rho_{A^{\prime} B}\right)\right] .
\end{aligned}
$$

This allows us to take advantage of another fundamental result of quantum information, the nonadditivity of $E_{f}\left(\rho_{A B}\right)$ [26]. Therefore, despite the additivity of the (relative) von Neumann entropy, we can state that the ability for Alice to perform global measurements can increase the amount of work Bob can distill; i.e., for some states $\rho_{A B}$ it will happen that

$$
W_{a}^{B \mid A}\left(\rho_{A B}\right)<W_{a, \infty}^{B \mid A}\left(\rho_{A B}\right) .
$$

However, for many simple classes of states, the above does not happen. For instance, in Appendix E [9], we explicitly calculate $W_{a}^{B \mid A}$ for the relevant family of isotropic states in arbitrary dimension and show its additivity over multiple copies.

Work of collaboration.-Let us now consider an arbitrary class of operations $\mathcal{O}$ on a thermodynamical system. We assume that $\mathcal{O}$ contains not only deterministic operations, but also the so-called quantum instruments, i.e., collections $\left\{\Phi_{i}\right\}_{i}$ of completely positive maps such that $\sum_{i} \Phi_{i}$ is trace preserving. Physically, the classical label $i$ will record the outcomes of the quantum measurements that have been made throughout the process, whereas $\operatorname{Tr} \Phi_{i}(\rho)$ represents the probability of the outcome $i$ occurring when the state $\rho$ is processed. In a bipartite setting, we can construct the associated set $\mathcal{O}_{c}^{B \mid A}$ of collaborative operations by concatenating in any of the following order: (1) instruments in $\mathcal{O}$ on $B$; (2) classical communication between Alice and Bob; and (3) arbitrary quantum operations on $A$. We can now define the associated work of collaboration in analogy with Eq. (2) as

$$
\begin{aligned}
W_{c}^{B \mid A}\left(\rho_{A B}\right):=\sup \{R E: & \\
& \lim _{n \rightarrow \infty} \inf _{\Lambda \in \mathcal{O}_{c}^{B \mid A}} \| \Lambda\left(\rho_{A B}^{\otimes n} \otimes|0\rangle\left\langle\left. 0\right|_{P} ^{\otimes[R n]}\right)-|1\rangle\left\langle\left. 1\right|_{P} ^{\otimes[R n]} \|_{1}=0\right\},\right.
\end{aligned}
$$

where it is understood that the battery $P$ pertains to Bob's system, and its Hamiltonian is again given by $H_{P}:=E|1\rangle\left\langle\left. 1\right|_{P}\right.$, with $E$ a free parameter.

By their very definition in Eq. (1), TO are intrinsically deterministic. Therefore, in the collaborative setting, there 
is no information Bob can send to Alice if he is restricted to TO, and the corresponding work of collaboration reduces to the regularized work of assistance as given in Eq. (7). To investigate the collaborative setting in greater detail, it is thus indispensable to expand Bob's allowed operations to the wider class of GP operations [7], that satisfy $\Lambda\left(\gamma_{B}\right)=\gamma_{B}$. This less restrictive framework crucially allows Bob to apply nondeterministic instruments $\left\{\Phi_{i}\right\}_{i}$, which are required to satisfy $\Phi_{i}\left(\gamma_{B}\right) \propto \gamma_{B}$ for all $i$. The outcome $i$ can then be communicated to Alice via the classical communication channel.

From now on, we will therefore consider the work of collaboration of Eq. (15) as defined for the collaborative set of operations $\mathrm{GP}_{c}^{B \mid A}$ corresponding to GP operations on Bob. It is clear that QT states of the form $\Gamma_{A B}=\sigma_{A} \otimes \gamma_{B}$, where $\sigma_{A}$ is arbitrary, can be generated for free even in the TO framework. Furthermore, it can be shown that these are all the states for which $W_{c}^{B \mid A}\left(\rho_{A B}\right)=0$. This suggests the following definition of the relative entropy of collaboration

$$
W_{r}^{B \mid A}\left(\rho_{A B}\right):=\frac{1}{\beta} \min _{\sigma_{A}} S\left(\rho_{A B} \| \sigma_{A} \otimes \gamma_{B}\right),
$$

where the minimization is taken over the set of QT states. In Appendix F [9], we explicitly demonstrate monotonicity of this function under the set of allowed operations. We also prove in Appendix F [9] that the minimization in (16) can be explicitly solved so as to give

$$
W_{r}^{B \mid A}\left(\rho_{A B}\right)=\frac{1}{\beta} S\left(\rho_{A B} \| \rho_{A} \otimes \gamma_{B}\right) .
$$

Simple algebraic manipulations allow us to recast this as

$$
W_{r}^{B \mid A}\left(\rho_{A B}\right)=\frac{1}{\beta}\left[S\left(\rho_{B} \| \gamma_{B}\right)+I\left(\rho_{A B}\right)\right],
$$

where $I\left(\rho_{A B}\right):=S\left(\rho_{A}\right)+S\left(\rho_{B}\right)-S\left(\rho_{A B}\right)$ is the mutual information quantifying total correlations between Alice and Bob.

Comparing measures of assistance.-Equation (18) suggests that the mutual information quantifies the amount by which the collaboration between the parties increases Bob's distillable work.

In fact, we are able to demonstrate in Appendix G [9] that $W_{r}^{B \mid A}$ provides an upper bound on the work of collaboration. We can also observe that since TO are a subset of GP operations, the work of collaboration is no smaller than the regularized work of assistance. This can also be deduced by comparing Eq. (9) with Eq. (18), and using the well-known fact that $C_{D}\left(\rho_{A B}\right) \leq I\left(\rho_{A B}\right)[21,27]$. Putting all together, we obtain that

$$
W_{a}^{B \mid A}\left(\rho_{A B}\right) \leq W_{a, \infty}^{B \mid A}\left(\rho_{A B}\right) \leq W_{c}^{B \mid A}\left(\rho_{A B}\right) \leq W_{r}^{B \mid A}\left(\rho_{A B}\right) .
$$

Recall from Eq. (14) that there can be a strict inequality between the two leftmost quantities in the above chain of inequalities. Concerning the two rightmost ones, quite interestingly, we find that the gap $W_{r}^{B \mid A}\left(\rho_{A B}\right)-$ $W_{a}^{B \mid A}\left(\rho_{A B}\right)$ is explicitly described by the quantum discord, a measure of the quantumness of the correlations between Alice and Bob [19,28]. Indeed, by comparing Eqs. (6) and (18), we find that

$$
\begin{aligned}
W_{r}^{B \mid A}\left(\rho_{A B}\right)-W_{a}^{B \mid A}\left(\rho_{A B}\right) & =\frac{1}{\beta}\left[I\left(\rho_{A B}\right)-J^{\rightarrow}\left(\rho_{A B}\right)\right] \\
& =: \frac{1}{\beta} D^{\rightarrow}\left(\rho_{A B}\right),
\end{aligned}
$$

where $D^{\rightarrow}\left(\rho_{A B}\right)$ is the quantum discord, quantifying the share of correlations lost between Alice and Bob as a consequence of a minimally disturbing measurement on Alice's side. This result shows that the work of collaboration can exceed the work of assistance by an amount bounded from above by the shared quantum correlations, measured by the discord $D^{\rightarrow}\left(\rho_{A B}\right)$. We note that recent works $[20,29]$ have suggested a protocol for explicitly distilling the work locked in quantum discord; however the operations considered are not TO. Other interpretations for the quantum discord in thermodynamical and related contexts have also been explored in the literature [22,30-32].

It is particularly instructive to analyze all the quantities appearing in Eq. (19) for the relevant case where Alice holds a purification of Bob's state, i.e., $\rho_{A B}=\phi_{A B}=$ $|\phi\rangle\left\langle\left.\phi\right|_{A B}\right.$. On the one hand, for a pure state $\phi_{A B}$ it is known $[19,21]$ that the Henderson-Vedral measure and distillable common randomness coincide with the local entropy of each subsystem, i.e., $J^{\rightarrow}\left(\phi_{A B}\right)=C_{D}\left(\phi_{A B}\right)=$ $S\left(\phi_{B}\right)$. Hence

$W_{a}^{B \mid A}\left(\phi_{A B}\right)=W_{a, \infty}^{B \mid A}\left(\phi_{A B}\right)=\frac{1}{\beta}\left[S\left(\rho_{B} \| \gamma_{B}\right)+S\left(\rho_{B}\right)\right]$,

implying that for an initial pure state the ability for Alice to perform global measurements over many copies gives no advantage in Bob distilling work. On the other hand, it is also elementary to verify that

$$
W_{r}^{B \mid A}\left(\phi_{A B}\right)=\frac{1}{\beta}\left[S\left(\rho_{B} \| \gamma_{B}\right)+2 S\left(\rho_{B}\right)\right] .
$$

Therefore, by comparing Eqs. (21) and (22), it is seen that for an initial pure state we demonstrate that relaxing the local operations from TO to GP maps might allow Bob to distill a bound quantity of work equal to the local entropy.

Conclusion.-In this Letter, we have fully characterized the task of assisted work distillation in the asymptotic scenario of quantum thermodynamics, addressing questions left open in [22,33]. In particular, we have introduced two relevant quantities of interest, the work of assistance and the work of collaboration. These quantities allowed us to investigate the possible advantage of local GP operations 
over TO and global measurements on a system; in particular, how GP operations may allow Bob to locally distill the work bound within the quantum correlations of the initial shared state.

Although it was shown that GP operations can provide an increase in distillable work, the explicit relationship between the work of assistance and the work of collaboration requires further investigation, as for the latter quantity only an upper bound was derived here. We further stress that our results only hold in the asymptotic limit. It would be interesting to investigate assisted work distillation in the single-shot regime to determine the role correlations play in work fluctuations. This could prove useful for nearterm technological applications.

The present analysis adds to the literature on assisted distillation of different quantum resources [6,33-37]. In particular, Refs. [33,36] studied the distillation of quantum coherence [38], rather than work, from Bob's system with the assistance of Alice. In that setting, Bob is limited to incoherent operations [39] while Alice can perform arbitrary local quantum operations, and the two parties can communicate classically. We can draw a comparison between the two settings, by noting that the additional quantity of resource that can be distilled from Bob's system thanks to Alice's assistance amounts to the entropy of Bob's reduced state in the case of coherence [33] and to the classical correlations shared between Alice and Bob in the case of work [Eq. (6)]. We can further observe how the hierarchy presented in Eq. (19) for assisted work distillation is analogous to the one derived in Ref. [33] for assisted coherence distillation, but the key role of quantum discord in bounding the gap between work of assistance and work of collaboration is only revealed in this Letter by comparing the power of different classes of local operations for Alice (TO versus GP). It would be meaningful to revisit the assisted coherence distillation framework by imposing additional physical constraints on Alice's operations, e.g., by adopting strictly incoherent operations [40] or TO, and hence exploiting the methods developed in this Letter for the characterization of other quantum resources.

Our findings could have implications for the understanding of the Szilard engine [41]. The latter is a simple physical model that demonstrates how information may be exploited in order to extract physical work. The relevance of this model was then understood in the context of information processing by Landauer [42]. Many recent works have discussed the application of a Szilard engine in quantum thermodynamics [43-49], deriving bounds for work extraction that are related to Eq. (18) [46-49] in a setting where a second party, historically entitled Maxwell's Demon, is in possession of a state correlated to the thermodynamic system. The converse setting, where correlations can be formed from initially uncorrelated states using thermal operations, has also been studied [50].
The results presented here provide further links between the fields of quantum information and thermodynamics. In particular, they demonstrate how highly studied measures of information provide us with an insight into the thermodynamics of correlations. These results both contribute to our knowledge of the fundamental nature of thermodynamics but also may become essential for the thermodynamic control of a quantum computer.

We are grateful to Harry Miller, Philippe Faist, Luis Correa, Carlo Maria Scandolo, Mark Whitworth, Ryuji Takagi, Francesco Plastina, and Paul Hollywood for helpful discussions. We acknowledge financial support from the European Research Council under the Starting Grant GQCOP (Grant No. 637352) and the EPSRC (Grant No. EP/N50970X/1).

*benjamin.morris@nottingham.ac.uk

†ludovico.lami@gmail.com

†gerardo.adesso@nottingham.ac.uk

[1] R. Alicki and R. Kosloff, arXiv:1801.08314.

[2] C. Elouard, D. A. Herrera-Martí, M. Clusel, and A. Auffèves, npj Quantum Inf. 3, 9 (2017).

[3] J. Goold, M. Huber, A. Riera, L. del Rio, and P. Skrzypczyk, J. Phys. A 49, 143001 (2016).

[4] D. Janzing, P. Wocjan, R. Zeier, R. Geiss, and T. Beth, Int. J. Theor. Phys. 39, 2717 (2000).

[5] F. G. S. L. Brandao, M. Horodecki, J. Oppenheim, J. M. Renes, and R. W. Spekkens, Phys. Rev. Lett. 111, 250404 (2013).

[6] D. P. DiVincenzo, C. A. Fuchs, H. Mabuchi, J. A. Smolin, A. Thapliyal, and A. Uhlmann, in Quantum Computing and Quantum Communications (Springer, New York, 1999), pp. 247-257.

[7] P. Faist, J. Oppenheim, and R. Renner, New J. Phys. 17, 043003 (2015).

[8] M. Horodecki and J. Oppenheim, Nat. Commun. 4, 2059 (2013).

[9] See Supplemental Material at http://link.aps.org/ supplemental/10.1103/PhysRevLett.122.130601 for proofs and technical derivations, which includes Refs. [10-17].

[10] M. Horodecki and P. Horodecki, Phys. Rev. A 59, 4206 (1999).

[11] C. King, IEEE Trans. Inf. Theory 49, 221 (2003).

[12] M. Hayashi, Quantum Information Theory: Mathematical Foundation, Graduate Texts in Physics (Springer, Berlin, Heidelberg, 2016).

[13] M. Fannes, Commun. Math. Phys. 31, 291 (1973).

[14] K. Audenaert, J. Phys. A 40, 8127 (2007).

[15] A. Winter, Commun. Math. Phys. 347, 291 (2016).

[16] V. Vedral and M. B. Plenio, Phys. Rev. A 57, 1619 (1998).

[17] A. Winter, Commun. Math. Phys. 347, 291 (2016).

[18] V. Narasimhachar and G. Gour, Phys. Rev. A 95, 012313 (2017).

[19] L. Henderson and V. Vedral, J. Phys. A 34, 6899 (2001).

[20] G. Manzano, F. Plastina, and R. Zambrini, Phys. Rev. Lett. 121, 120602 (2018). 
[21] I. Devetak and A. Winter, IEEE Trans. Inf. Theory 50, 3183 (2004).

[22] J. Oppenheim, M. Horodecki, P. Horodecki, and R. Horodecki, Phys. Rev. Lett. 89, 180402 (2002).

[23] M. Koashi and A. Winter, Phys. Rev. A 69, 022309 (2004).

[24] C. H. Bennett, G. Brassard, S. Popescu, B. Schumacher, J. A. Smolin, and W. K. Wootters, Phys. Rev. Lett. 76, 722 (1996).

[25] P. M. Hayden, M. Horodecki, and B. M. Terhal, J. Phys. A 34, 6891 (2001)

[26] M. B. Hastings, Nat. Phys. 5, 255 (2009).

[27] I. Devetak and T. Berger, Phys. Rev. Lett. 87, 197901 (2001).

[28] H. Ollivier and W. H. Zurek, Phys. Rev. Lett. 88, 017901 (2001).

[29] G. Francica, J. Goold, F. Plastina, and M. Paternostro, npj Quantum Inf. 3, 12 (2017).

[30] W. H. Zurek, Phys. Rev. A 67, 012320 (2003).

[31] P. Liuzzo-Scorpo, L. A. Correa, R. Schmidt, and G. Adesso, Entropy 18, 48 (2016).

[32] G. Adesso, T. R. Bromley, and M. Cianciaruso, J. Phys. A 49, 473001 (2016).

[33] E. Chitambar, A. Streltsov, S. Rana, M. N. Bera, G. Adesso, and M. Lewenstein, Phys. Rev. Lett. 116, 070402 (2016).

[34] M. Horodecki, K. Horodecki, P. Horodecki, R. Horodecki, J. Oppenheim, A. SenDe, U. Sen, Phys. Rev. Lett. 90, 100402 (2003).

[35] I. Devetak, Phys. Rev. A 71, 062303 (2005).
[36] B. Regula, L. Lami, and A. Streltsov, Phys. Rev. A 98, 052329 (2018).

[37] A. Streltsov, S. Rana, M. N. Bera, and M. Lewenstein, Phys. Rev. X 7, 011024 (2017).

[38] A. Streltsov, G. Adesso, and M. B. Plenio, Rev. Mod. Phys. 89, 041003 (2017).

[39] T. Baumgratz, M. Cramer, and M. B. Plenio, Phys. Rev. Lett. 113, 140401 (2014).

[40] A. Winter and D. Yang, Phys. Rev. Lett. 116, 120404 (2016).

[41] L. Szilard, Z. Phys. 53, 840 (1929).

[42] R. Landauer, IBM J. Res. Dev. 5, 183 (1961).

[43] S. W. Kim, T. Sagawa, S. De Liberato, and M. Ueda, Phys. Rev. Lett. 106, 070401 (2011).

[44] M. H. Mohammady and J. Anders, New J. Phys. 19, 113026 (2017).

[45] N. Cottet, S. Jezouin, L. Bretheau, P. Campagne-Ibarcq, Q. Ficheux, J. Anders, A. Auffèves, R. Azouit, P. Rouchon, and B. Huard, Proc. Natl. Acad. Sci. U.S.A. 114, 7561 (2017).

[46] J. J. Park, K.-H. Kim, T. Sagawa, and S. W. Kim, Phys. Rev. Lett. 111, 230402 (2013).

[47] W. H. Zurek, in Frontiers of Nonequilibrium Statistical Physics (Springer, New York, 1986), pp. 151-161.

[48] D. Reeb and M. M. Wolf, New J. Phys. 16, 103011 (2014).

[49] T. Sagawa and M. Ueda, Phys. Rev. Lett. 100, 080403 (2008).

[50] V. Narasimhachar, J. Thompson, J. Ma, G. Gour, and M. Gu, Phys. Rev. Lett. 122, 060601 (2019). 